\section{耍 Heighten Science \\ P U B L I C I T I O N S Corporation \\ ISSN \\ 2576-9529}

\title{
Causes of hospital admission of chronic kidney disease patient in a tertiary kidney care hospital
}

\author{
Salman Imtiaz ${ }^{1 *}$, Ruqaya Qureshi², Amna Hamid ${ }^{3}$, Beena \\ Salman ${ }^{4}$, Murtaza F Drohlia ${ }^{5}$ and Aasim Ahmad ${ }^{6}$
}

${ }^{1}$ Professor \& Consultant, Nephrologist, Department of Nephrology, The Kidney Centre, Post Graduate Training Institute, Karachi, Pakistan

${ }^{2}$ Assistant Professor \& Consultant Nephrologist, Department of Nephrology, The Kidney Center Hospital, 197/9, Rafiqui Shaheed Road, Karachi, Pakistan

${ }^{3}$ Resident Nephrology, The Kidney Center Hospital, 197/9, Rafiqui Shaheed Road, Karachi, Pakistan

${ }^{4}$ Research Specialist, Post Graduate Training Institute. The Kidney Center Hospital, 197/9, Rafiqui Shaheed Road, Karachi, Pakistan

${ }^{5}$ Assistant Professor \& Consultant, Nephrologist, The Kidney Center Hospital, 197/9, Rafiqui Shaheed Road, Karachi, Pakistan

${ }^{6}$ Professor, Aasim Ahmad Chairperson Bioethics Group, Director Master of Bioethics (MBeth) Hon. Senior Lecturer, Aga Khan University, Dean \& Chief Nephrologist, The Kidney Center Hospital, 197/9, Rafiqui Shaheed Road, Karachi, Pakistan

\section{*Address for Correspondence: Dr. Salman Imtiaz; Professor \& Consultant, Nephrologist, Department of Nephrology, The Kidney Centre, Post Graduate Training Institute, Karachi, Pakistan, Tel: 021-3566 1000 (10 Lines) (Ext. 253); Mobile: (092)03042007270; Fax: 021-3566 1040, 021-3566 1050; Email: salman_imtiaz@hotmail.com salman.imtiaz@tih.org.pk}

Submitted: 13 May 2019 Approved: 20 June 2019 Published: 21 June 2019

Copyright: ๑ 2019 Imtiaz S, et al. This is an open access article distributed under the Creative Commons Attribution License, which permits unrestricted use, distribution, and reproduction in any medium, provided the original work is properly cited

Keywords: Chronic kidney disease (CKD); Cardiovascular disease (CVD); Hospitalization; Urinary Tract infection (UTI)

Check for updates

\section{Abstract}

Background: Patients with chronic kidney disease (CKD) are at the risk of increase hospital admission as compared to the general population, due to various reasons. They have increased vulnerability to cardiovascular diseases (CVD) as well as infections, therefore they usually got admit in health care units due to various reasons. The causes of hospitalization in CKD patients in this part of the world are not studied well.

Methods: This cross sectional study was conducted in The Kidney Centre Post Graduate Training Institute (TKC-PGTI) of Karachi. Variables included in the study were age, gender, are of residence, ethnicity, smoking status and level of education. Comorbid conditions like causes of CKD and causes of hospitalization. Data analysis performed by using software IBM SPSS 21.

Results: Total of 269 patients were enrolled in our study. The male 148(55\%), mean age was 55 years. The most common cause of hospitalization in our population was infection $148(55 \%)$ and urinary tract infection (UTI) was the most common site of infection 55 (20\%) followed by sepsis of unknown origin $29(10.8 \%)$. Cardiovascular events like volume overload $32(11.4 \%)$ and acute coronary syndrome $20(7.4 \%)$ were the second most important cause of hospitalization.

Conclusion: Hospitalization of CKD patients is high, and in our population infection is found to be the leading cause of hospital admission. Infection originating from urinary tract is more common in all stages of CKD patients, while blood born infection originating from double lumen (DL) dialysis catheter or arteriovenous fistula (AVF) cannulation is more common in dialysis population. Cardiovascular events, both acute coronary syndrome and pulmonary edema due to volume overload followed the infections.

\section{Introduction}

CKD is prevalent, and its incidence is rising in all parts of the world [1]. The outcome of the patients suffering with CKD is poor. They have high risk of experiencing noninfectious as well as infectious diseases, for example the risk of CVD is high as compared with the general population and on the other hand they also have higher

How to cite this article: Imtiaz S, Qureshi R, Hamid A, Salman B, Drohlia MF, et al. Causes of hospital admission of chronic kidney disease patient in a tertiary kidney care hospital. J Clini Nephrol. 2019; 3: 100-106. https://doi.org/10.29328/journal.jcn.1001033 
propensity to acquire several infections [2,3]. Risk of hospitalization is high among the CKD population, there are various etiologies but mostly the cause is cardio vascular events [4]. This exaggerated risk is difficult to explain by the traditional risk factors. The phenomena of "reverse epidemiology" of the risk factors, was observed in CKD patients. Along with conventional risk factors for CVD like obesity, hypertension, and hypocholesteremia which predict mortality in general population, a declining body weight, low blood pressure and low cholesterol also push these patients to have a cardiac event. Therefore, the prediction for future cardiovascular event, management of ongoing CVD should take in consideration while combating with heart disease in CKD patients $[5,6]$.

Other than cardiovascular phenomenon which is identified as leading cause of hospitalization, infections are recognized as second most important cause of increased morbidity and mortality in CKD patients [7]. The reason of this is thought to be due to the disturbance of hormones and accumulation of metabolic end products. This uremic milieu badly affects the bones, blood vessels and immune system. This modified immune system is a well-known clinical consequence of CKD of high advance stages, observed by a markedly high rate of infection and infection related mortality in dialysis population $[8,9]$. Bacteremia in dialysis patients is attributed to the vascular access [10]. Patients with less sever disease kidney diseases are also at a high risk of infection related complications [11]. It is uncertain if reduced kidney function increases the risk of acquiring infection in community. In a large case control study, CKD was recognized as a risk factor for incidence of community acquired pneumonia in primary care [12].

Hospitalization conceded as major burden for patients with CKD. On average, End-Stage Renal Disease (ESRD) patients are admitted to the hospital nearly twice a year, and about $30 \%$ have an unplanned re-hospitalization within 30 days following discharge. Given the disruption of everyday life stemming from dialysis treatment, hospital admission and readmission additionally compromised patients' wellbeing and quality of life and are associated with adverse clinical outcome. Furthermore in these patients, treatment represents a significant societal and financial burden, accounting for approximately $40 \%$ of total Medicare expenditure for dialysis patients [13].

In this study we evaluated the causes of hospital admission in CKD patients in context with age, gender, stages of CKD, economical status and site of infection.

\section{Material and Methods}

This cross sectional study was done at the Kidney Center Post Graduate Training Institute (TKC-PGTI). Ethical review was taken from the Ethical review committee of the Kidney center post graduate medical institute with Reference No. 23-NEPH032015. CKD was defined, according to Kidney Disease Improving Global Outcomes (KDIGO) position statement, as kidney damage or glomerular filtration rate (GFR) $<60$ $\mathrm{mL} / \mathrm{min} / 1.73 \mathrm{~m} 2$ for 3 months or more, irrespective of cause. And then according to the GFR they were classified in five stages of CKD Stage 1: glomerular filtration rate (GFR) > $90 \mathrm{ml} / \mathrm{min}$. Stage 2: GFR 60-89 ml/min, Stage 3a: GFR45-49 ml/min, Stage 3b: GFR 30-45 ml/min, Stage 4: GFR 15-29 ml/min, Stage 5: GFR< $15 \mathrm{ml} / \mathrm{min}$. We included all CKD patients, 18 years of age and above who were already admitted at the time of study initiation. Patients who visited emergency department (ED) or outpatient department (OPD) of Kidney center Karachi during the study period and who were advised for admission during the visit. We excluded Patients with acute renal failure and those who electively admitted for renal biopsy or angioacces formation (AV Fistula).

Cases who were being advised for admission were followed up in the ward for further screening and data collection. If found eligible they were offered informed written consent for inclusion into the study. Those who gave consent to participate 
were asked for providing a 15-20 minutes interview for the data collection. Informed consent was translated in Urdu language.

All patients who got admitted in the TKC-PGTI in the 6-month period from January 2016 to 30th June 2016 were enrolled for the research. Variables included in the study were gender, age, marital status, area of residence, ethnicity, smoking status, and level of education. Comorbid conditions, causes of CKD and cause of hospitalization were also analyzed

Data analyses were performed by using software IBM SPSS license version 21. Cleaning and coding of the data were done prior to analysis. Continuous variables were described in terms of median with IQR while categorical variables were mentioned in frequencies with percentages.

\section{Results}

Total of 269 patients were enrolled in our study, in which 148(55\%) were male. Most of our patient's population was married (82.9\%) and majority of them belonged to middle socio economic group (52.4\%). Uneducated patients were higher in number 71(26.4\%).Among 121 female, 115 were house wives. The majority of the patients were residents of Karachi [192(71.4\%)] and in the same manner the highest number of patients [116(43.1)] were Urdu speaking. Our most of the patients were never smoker 207(77\%) (Table 1). Patient's clinical parameters showed in table 2.

CKD patients had many co morbid conditions, majority of the patients were diabetic and among different stages of CKD, however stage $V$ was most prevalent 118(43.9) while 97(36.1) patients were on hemodialysis (HD) (Table 3).

Among different causes of hospitalization in CKD patients the most common

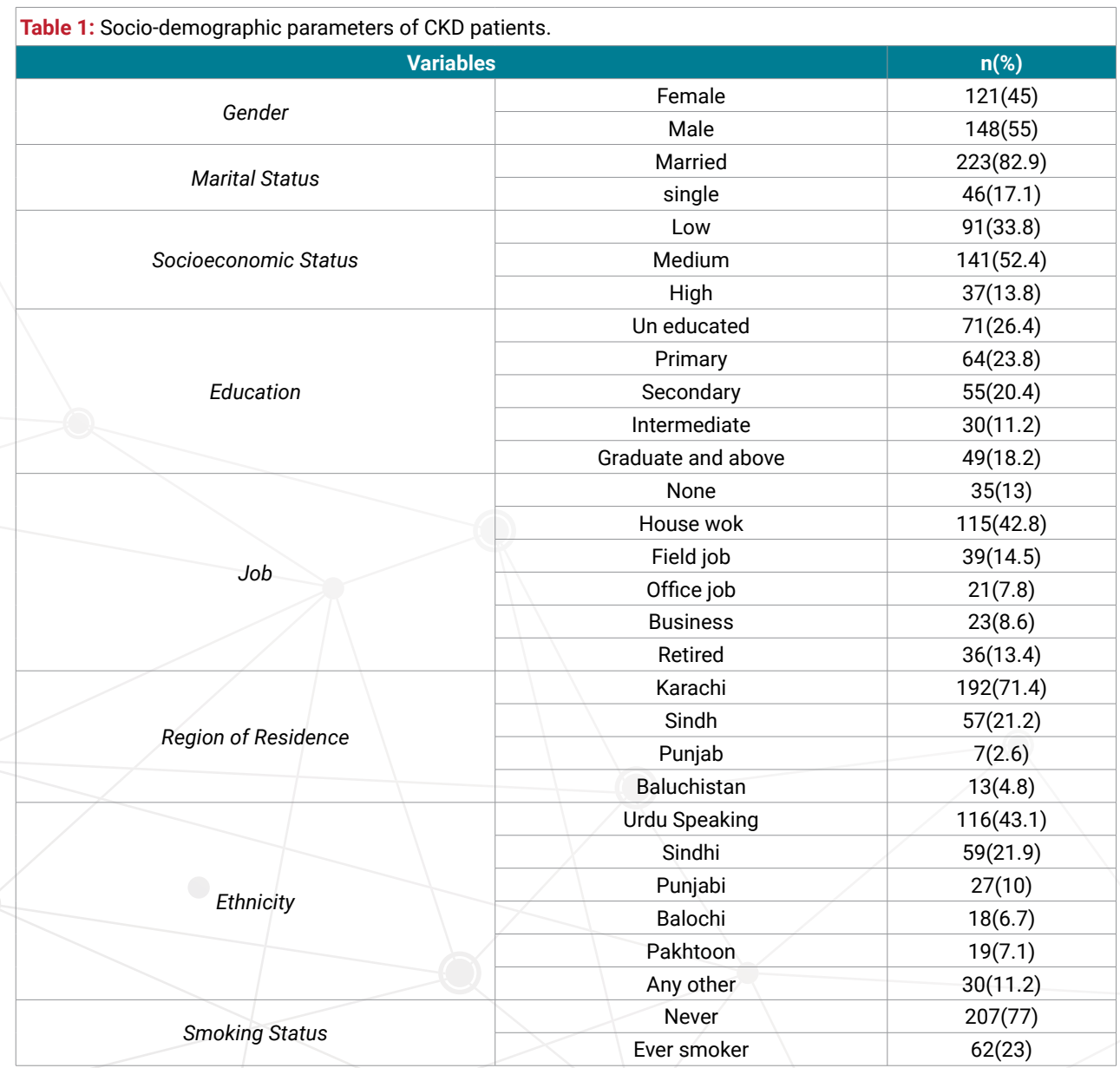


Table 2: Clinical and laboratory parameters of CKD Patients.

\begin{tabular}{|c|c|c|c|}
\hline Variables & Median, IQR & Minimum & Maximum \\
\hline Age & 55,19 & 18 & 81 \\
\hline Weight & $60.9,19.7$ & 23 & 147 \\
\hline Systolic BP & 130,40 & 50 & 220 \\
\hline Diastolic BP & 80,20 & 30 & 120 \\
\hline Hemoglobin & $9.3,2.8$ & 3.1 & 31 \\
\hline Creatinine & $6.8,5.6$ & 0.5 & 38.3 \\
\hline Total leucocyte count & $10.8,6.3$ & 2.9 & 4.5 \\
\hline Albumin & $3.3,0.7$ & 1.2 & 439 \\
\hline Urea & $136,111.2$ & 20 & 241 \\
\hline CKD: Chronic Kidney Disease; BP: Blood Pressure & $18.7,28.7$ & 6 & \\
\hline
\end{tabular}

Table 3: Comorbid condition associated with the patients along with Chronic kidney disease.

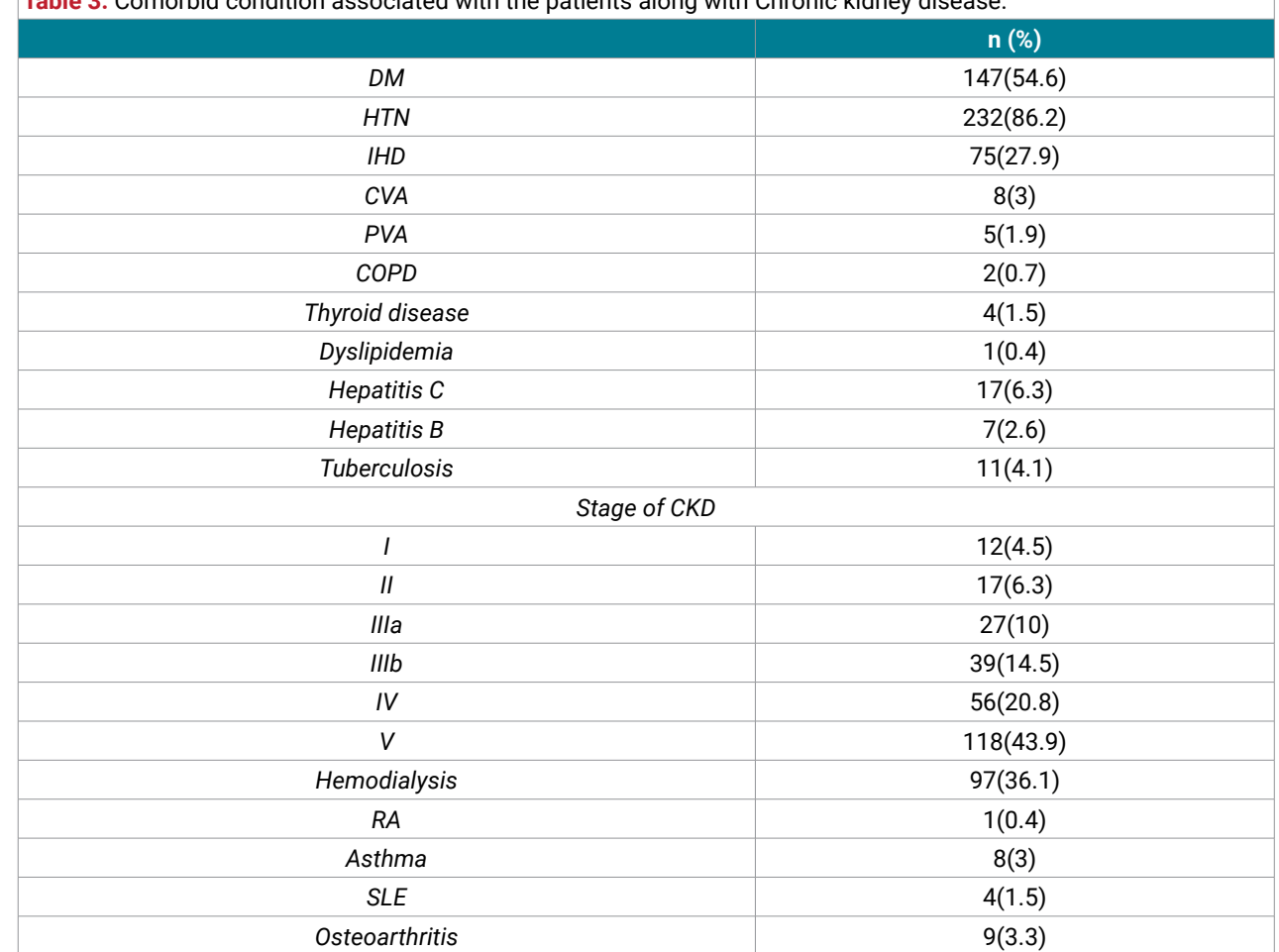

DM: Diabetes Mellitus; HTN: Hypertension; IHD: Ischemic Heart Disease; CVA: Cerebrovascular Accident; PVA: Peripheral Vascular Disease; COPD: Chronic Obstructive Pulmonary Disease; RA: Rhumatoid Arthritis; SLE: Systemic Lupus Erythemetosis

cause of hospitalization was infection 148(55\%), mostly originated from urinary tract 55 (20.4\%), and followed by sepsis of unknown origin, 29(10.8\%). Sepsis due to central venous line was $10 \%$, while respiratory tract infection was $21(7.8 \%)$. Among noninfectious causes of hospitalization cardiac conditions were the leading one, majority of the patients presented with volume overload $32(11.9 \%)$ and acute coronary syndrome $[20(7.4 \%)]$ (Table 4$)$.

\section{Discussion}

This is the first study conducted to see hospital admission in CKD patients in this country, we didn't find any other study, even in the general medicine discipline. This study showed that infection is the most common cause of hospital admission in this population followed by cardiovascular events. As we discussed earlier infection is considered as second leading cause of hospitalization and death among patients undergoing HD even in North America. As we discussed earlier CKD patients have substantially more propensity to be hospitalized then those who have normal kidney function [9]. High burden of community acquired blood-born infections is recognized in developing countries in non CKD population [14]. The vulnerability to acquire 


\begin{tabular}{|c|c|}
\hline \begin{tabular}{|c|} 
Table 4: Causes of hospital admission in CKD Patients. \\
\hline Causes of hospitalization \\
\hline Infectious causes
\end{tabular} & $\mathbf{N}(\%)$ \\
\hline UTI & $55(20.4)$ \\
\hline Sepsis of unknown origin & $29(10.8)$ \\
\hline DL catheter sepsis & $27(10)$ \\
\hline RTI & $21(7.8)$ \\
\hline Gastro Intestinal infection & $16(5.9)$ \\
\hline Noninfectious causes & $121(44.0)$ \\
\hline Fluid Overload(CCF) & $32(11.9)$ \\
\hline Uremic Symptoms & $28(10.4)$ \\
\hline Acute Coronary Syndrome & $20(7.4)$ \\
\hline Volume Depletion & $10(3.7)$ \\
\hline Acute on Chronic with no obvious cause & $8(3.0)$ \\
\hline Metabolic Acidosis & $8(3.0)$ \\
\hline Electrolyte Imbalance & $8(3.0)$ \\
\hline Neurological Problems & $5(1.9)$ \\
\hline PVD / DVT & $2(0.7)$ \\
\hline Total & $269(100.0)$ \\
\hline
\end{tabular}

CKD: Chronic Kidney Disease; UTI: Urinary Tract Infection; DL: Double Lumen Catheter; RTI: Respiratory Tract Infection; PVD/DVT: Peripheral Vascular Disease/Deep Venous Thrombosis; CCF: Congestive Cardiac Failure

infections increases in CKD population due to reduce immunological response, frequent exposure to hospital environment etc. We also found infection as a leading cause of hospitalization in our population. Among different systems affected with the infection, UTI was the most common cause of hospital admission in all stages of CKD. While central venous catheter was a common cause of infection in HD patients, in whom HD was started recently or in chronic HD patients in which vascular access is not working Hospitalization due to respiratory tract infection was not common in our population as mentioned in other studies. Among people with CKD there is paucity of randomized control trial which examined the incidence of infection according to the GFR stage specific infection rates. It is evident from the epidemiological studies that Pneumonia is the leading cause of infection related complication that make the CKD patients to get admitted in hospital $[15,16]$, while line related sepsis is the most common cause among HD patients [17]. There are many factors which determined the site of infection in CKD patients among different geographical areas, for example high incidence of COPD and kidney stone disease in any population, increases the incidence of pulmonary and genitourinary tract infection respectively.

Modalities of HD, for example in-center versus home HD also affect the reason for hospitalization. An interesting finding was observed in a cohort study on two groups of patients, one with daily home HD and other in center thrice weekly dialysis. Hospitalization due to cardiovascular instability in daily home HD population was less as compared with thrice weekly in center HD possibly due to better removal of water in daily dialysis patients. On the contrary, the rate of infection related hospitalization was found as high in daily home HD patients as compared to in center thrice weekly HD patients possibly due to frequent cannulation [18]. In our study population $32(11.9 \%)$ patients were admitted with congestive cardiac failure and $20(7.4 \%)$ of the patients were admitted with acute coronary syndrome. This number of patients with acute coronary syndrome might be high if we include those patients who were referred to tertiary cardiology hospital from emergency department of the TKC-PGTI for urgent coronary intervention due to unavailability of intervention cardiology at TKC-PGTI. Same increasing trends of cardiovascular hospitalization among CKD patients were found both in dialysis [19] and pre-dialysis patients [20].

In conclusion, there are various reasons for hospitalization of CKD patients, and in our population infection is found to be the leading cause of hospital admission. Infection originating from urinary tract is more common in all stages of CKD patients, 
while blood born infection originating from DL dialysis catheter or AVF sssssss is more common in dialysis population. Cardiovascular events, both acute coronary syndrome and pulmonary edema due to volume overload followed the infections.

\section{Conclusion}

Hospitalization of CKD patients is outrageous and there are different causes of hospitalization in these patients, UTI, DL related sepsis and cardiovascular causes are most frequent causes of admission.

\section{Acknowledgements}

The author acknowledges his colleagues for their support and cooperation.

We acknowledged Ms. Yumna Maheen and Anees Badar Soomro for his support in collecting and arranging the data and for his help in preparing and arranging the manuscript.

\section{References}

1. Hill NR, Fatoba ST, Oke JL, Hirst JA, O'Callaghan CA, et al. Global prevalence of chronic kidney disease - A systemic review and Mata-Analysis. Plos On. 2016; 11: e0158765. Ref.: http://bit.ly/2WWdMFc

2. Culleton BF, Larson MG, Wilson PW, Evans JC, Parfrey PS, et al. Cardiovascular disease and mortality in a community-based cohort with mild renal insufficiency. Kidney int. 1999; 56: 2214-9. Ref.: http://bit.ly/2KtIX9m

3. Naqvi SB, Collins AJ. Infectious complications in chronic kidney disease. Adv Chronic Kidney Dis. 2006; 13:199-204. Ref.: http://bit.ly/31MPL7b

4. Tonelli M, Wiebe N, Culleton B, House A, Rabbat C, et al. Chronic kidney disease and mortality risk: A systematic review. J Am Soc Nephrol. 2006; 17: 2034-2047. Ref.: http://bit.ly/2RrFhpf

5. Kalantar-Zadeh K, Block G, Humphreys MH, Kopple JD. Reverse epidemiology of cardiovascular risk factors in maintenance dialysis patients. Kidney int. 2003; 63: 793-808. Ref.: http://bit.ly/2XnOoN7

6. Kopple JD. The phenomenon of altered risk factor patterns or reverse epidemiology in persons with advanced chronic kidney failure. Am J Clin Nutr. 2005; 81: 1257-1266. Ref.: http://bit.ly/2WYCGco

7. Sarnak MJ, Jaber BL. Mortality caused by sepsis in patients with end-stage renal disease compared with the general population. Kidney Int. 2000; 58: 1758-1764. Ref.: http://bit.ly/2RsIntd

8. Laurin LP, Harrak H, Elftouh N, Ouimet D, Vallée M, et al. Outcomes of infection-related hospitalization according to dialysis modality. Clin J Am Soc Nephrol. 2015: 10; 817-824. Ref.: http://bit.ly/2Rshjdn

9. Dalrymple LS, Mu Y, Romano PS, Nguyen DV, Chertow GM, et al. Outcomes of infection-related hospitalization in Medicare beneficiaries receiving in-center hemodialysis. Am J Kidney Dis. 2015; 65: 754-762. Ref.: http://bit.ly/2Zz25pV

10. Ishani A, Collins AJ, Herzog CA, Foley RN. Septicemia, access and cardiovascular disease in dialysis patients: the USRDS Wave 2 study. Kidney Int. 2005; 68: 311-318. Ref.: http://bit.ly/2N3oAlv

11. Dalrymple LS, Katz R, Kestenbaum B, de Boer IH, Fried L, et al. The risk of infection-related hospitalization with decreased kidney function. Am J Kidney Dis. 2012; 59: 356-363. Ref.: http://bit.ly/2IYACr3

12. Vinogradova $Y$, Hippisley-Cox J, Coupland C. Identification of new risk factors for pneumonia: population-based case-control study. Br J Gen Pract. 2009; 59: 329-338. Ref.: http://bit.ly/2NOfnu8

13. Saran R, Robinson B, Abbott KC, Agodoa LY, Albertus P, et al. US Renal Data System 2016 Annual Data Report: epidemiology of kidney disease in the United States. Am J Kidney Dis. 2017; 69: 7-8. Ref.: http://bit.ly/2XoKljr

14. Deen J, von Seidlein L, Andersen F, Elle N, White NJ, et al. Community-acquired bacterial bloodstream infections in developing countries in south and southeast Asia: a systematic review. Lancet Infect Dis. 2012; 12: 480-487. Ref.: http://bit.ly/2ZGelF7

15. Viasus D, Garcia-Vidal C, Cruzado JM, Adamuz J, Verdaguer R, et al. Epidemiology, clinical features and outcomes of pneumonia in patients with chronic kidney disease. Nephrology Dialysis Transplantation. 2011; 26: 2899-2906. Ref.: http://bit.ly/2Ku0duS

16. Chou CY, Wang SM, Liang CC, Chang CT, Liu JH, et al. Risk of Pneumonia Among Patients With 
Chronic Kidney Disease in Outpatient and Inpatient Settings: A Nationwide Population-Based Study. Medicine. 2014; 93: 1-4. Ref.: http://bit.ly/2L5COj9

17. Di Napoli A, Pezzotti P, Di Lallo D, Tancioni V, Papini P, et al. Determinants of hospitalization in a cohort of chronic dialysis patients in central Italy. J Nephrol. 2005; 18: 21-29. Ref.: http://bit.ly/2Xv15FK

18. Weinhandl ED, Nieman KM, Gilbertson DT, Collins AJ. Hospitalization in daily home hemodialysis and matched thrice-weekly in-center hemodialysis patients. Am J Kidney Dis. 2015; 65: 98-108. Ref.: http://bit.ly/2WVIQq2

19. Rocco MV, Soucie JM, Reboussin DM, McClellan WM. Risk factors for hospital utilization in chronic dialysis patients. Southeastern Kidney Council (Network 6). J Am Soc Nephrol. 1996; 7: 889-896. Ref.: http://bit.ly/2XoucdX

20. Holland DC, Lam M. Predictors of hospitalization and death among predialysis patients: a retrospective cohort study. Nephrol Dial Transplant. 2000; 15: 650-658. Ref.: http://bit.ly/2XsKGSi 